\title{
The Connection between Classical Be Stars and Herbig Ae/Be Stars
}

\author{
T. Böhm ${ }^{1}$ and L.A. Balona ${ }^{2}$ \\ ${ }^{1}$ Laboratoire Astrophysique, Observatoire Midi-Pyrenees, 14 av Edouard \\ Belin, F - 31400 Toulouse, France \\ ${ }^{2}$ South African Astronomical Observatory, P.O. Box 9, Observatory \\ 7935, Cape Town, South Africa.
}

\begin{abstract}
.
We use a phenomenological approach to derive new constraints on the mass loss mechanisms working in the classical $\mathrm{Be}$ and the Herbig $\mathrm{Ae} / \mathrm{Be}$ stars, which represent two groups of stars located very nearby in the HR diagram. We discuss observational similarities of classical Be and Herbig Be stars on one side, and stress, on the other side, the major differences between Herbig Ae and Herbig Be stars. For further details and a detailed bibliography, see Balona \& Böhm (2000). A more quantitative approach will be needed and is deferred to future work.
\end{abstract}

\section{Introduction}

Classical Be stars are normal B dwarfs or giants showing emission in the Balmer lines. In our galaxy, approximately $15 \%$ of early B stars are Be stars. Most of them are photometrically and spectroscopically variable, particularly in the Balmer lines. The emission is strongest in $\mathrm{H} \alpha$ which in most stars has a doublepeak profile. The broadened spectral lines show that the Be stars are rapid rotators with a mean value $v \sin i \approx 250 \mathrm{~km} \mathrm{~s}^{-1}$. The outflow velocities observed in the hydrogen lines are between several tens up to as much as $150 \mathrm{~km} \mathrm{~s}^{-1}$. Circumstellar disks have been imaged, and the the line formation region has been shown to extent to 5-10 stellar radii (Quirrenbach et al. 1997). Despite much speculation, the origin of the mass loss is still unknown.

The Herbig $\mathrm{Ae} / \mathrm{Be}$ stars constitute a very inhomogeneous group of premain sequence early B to late-A stars of intermediate mass. They are the high mass counterparts of the T Tauri stars. According to Herbig's selection criteria, they must be of spectral type A or earlier, show emission lines, be located in obscured regions with a reflection nebula in the vicinity. Additional criteria concerning their position in the HR diagram (above the main sequence) as well as the presence of a strong IR excess, have been added to the original list. They ensure that these are very young stars in the contracting phase towards the ZAMS. Herbig Ae/Be stars show outflows with mass loss rates of about $10^{-8}$ to $10^{-6} \mathrm{M}_{\odot} \mathrm{yr}^{-1}$, they are fast rotators, with a mean $v \sin i \approx 100 \mathrm{~km} \mathrm{~s}^{-1}$ (Böhm \& Catala 1995, hereafter BC). The Balmer lines show a variety of profiles, including $\mathrm{P}$ Cygni, double-peak and single-peak profiles. The likely presence of a 
corona and chromosphere is supported by observations in the far-UV and X-ray regions. Moreover, some Herbig stars show the presence of jets, bipolar outflows and accretion.

The presence or absence of circumstellar disks is a controversial question. This can be resolved by proposing an evolutionary scenario in which the deeplyembedded stars with high extinction, jets, bipolar outflows, asymmetric forbidden emission lines, very strong IR excesses, and the probable presence of disks suggested by polarization measurements, would be the young Herbig stars very close to their progenitor material. The more naked, isolated stars, without jets or bipolar outflows, with weaker IR excesses and lower extinctions constitute the more evolved Herbig stars. In this paper, we limit our discussion to this latter group of stars which, for the most part, have lost the original progenitor material and do not have a significant circumstellar accretion disk.

\section{Observational similarities and differences}

\subsection{Fraction}

Classical Be stars constitute less than $15 \%$ of normal B-type stars. Herbig Ae/Be stars constitute less than $4 \%$ of the A- and B-type PMS stars. These values are representative of our Galaxy only.

\subsection{Spectral energy distribution}

Classical Be stars have weak near-IR excesses. The significant IR excesses of Herbig Ae/Be stars have been classified in three groups (Hillenbrand et al. 1992). The IR excesses of group III objects resemble very much those found in the SEDs of classical Be stars, and are, moreover, found only around Herbig Be stars.

\subsection{H $\alpha$ line profiles}

Classical Be stars and Herbig Be stars show a very similar distribution of $\mathrm{H} \alpha$ line profiles: approximately one-third of these stars have a single peak profile, and $2 / 3$ a double peak profile. P Cygni profiles in $\mathrm{H} \alpha$ are observed almost only in late B- to early A-type Herbig stars. In these late-type Herbig stars, 35 percent have P Cygni profiles, 17 percent single-peak and 49 percent double-peak profiles.

\subsection{Rotation}

Classical Be stars are the fastest rotators among the B-type stars. Taking into consideration the fact that Herbig stars are still contracting, and that they will spin up during this phase, the Herbig stars are also fast rotators $(v \sin i \approx 110$ $\mathrm{km} \mathrm{s}^{-1}$ for Herbig Be stars and $\approx 90 \mathrm{~km} \mathrm{~s}^{-1}$ for Herbig Ae stars)(BC). Some very fast rotators can be found among the Herbig Be stars. It should be noted, however, that these $v \sin i$ values for Herbig Ae/Be stars are based on a rather small statistical sample which has a large scatter.

\subsection{Photometric variability}

While most classical Be stars show photometric variability, the amplitude generally decreases with decreasing effective temperature. Amplitudes are generally 


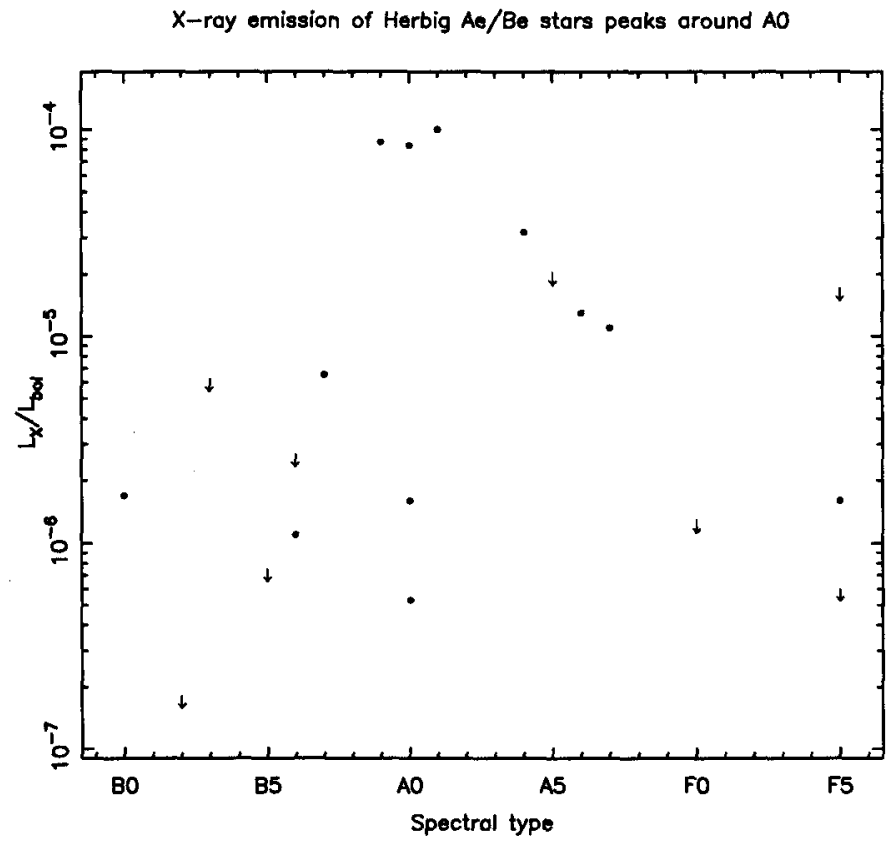

Figure 1. Fig.1. X-ray emission versus bolometric luminosity as a function of spectral type. Arrows indicate upper limits.

less than 0.1 mag. Herbig Be stars show very similar behaviour; their amplitudes are generally smaller than 0.5 mag. Herbig Ae stars are very different in their photometric variability. The amplitude of the variations lies mostly in the range 0.5 to 3 mag.

\subsection{Outflow velocities and winds}

Classical Be stars and Herbig Be stars show slow outflow velocities, generally less than several tens of $\mathrm{km} \mathrm{s}^{-1}$. However, highly-ionized wind tracers in classical Be stars show $P$ Cygni profiles with velocities up to $1000 \mathrm{~km} \mathrm{~s}^{-1}$. A dense, equatorial disk structure is responsible for the $\mathrm{H} \alpha$ profile. The radiativelydriven stellar wind is confined to higher latitudes. Herbig Ae stars seem to have only a spherically symmetric wind, indicated by $\mathrm{P}$ Cygni profiles with blue-edge velocities of 300 to $500 \mathrm{~km} \mathrm{~s}^{-1}$.

\subsection{Efficiency of emission of non-photospheric activity tracers}

In Herbig stars, the X-ray flux increases with effective temperature. However, there is a marked change around spectral class $A 0$, when there is a sudden increase of the X-ray emissivity, expressed as the ratio of the X-ray flux to the bolometric luminosity (Fig. 1). This same behaviour is also present in many other non-photospheric lines, where the ratio of the non-photospheric emission versus the bolometric luminosity of the star increased strongly around $\mathrm{A} 0(\mathrm{BC})$. 


\subsection{Magnetic fields}

Classical Be stars show short term, erratic, line profile variations, in some cases there is evidence for optical, UV and X-ray flaring. Moreover, strong indications of co-rotating gas clouds suspended above the photosphere recently been discovered. Rotational modulation of spectral lines in Herbig Ae/Be stars has long been accepted and confirmed on several different data sets. Very recently, the first direct detection of a weak magnetic field by spectropolarimetry has been performed. The inferred averaged longitudinal magnetic field is approximately 50 gauss (Donati et al. 1997). Taking into account all these indications, it seems very likely that there are indeed magnetic fields in both groups of stars. It is, of course, very important to understand the role of magnetic fields in photospheric activity, in the stellar wind and possibly as the cause of the Be phenomenon itself. To better understand the origin, strength and structure of a presumed magnetic field, is of major importance. It is true that generation of magnetic fields in stars without convective envelopes cannot be understood by the classical solar dynamo mechanism; a problem which needs to be addressed for further progress.

Two major conclusions of this section can be derived: (i) classical Be stars and Herbig Be stars have many common observational properties, and (ii) in many respects, the Herbig Ae stars show very different behaviour from the Herbig Be/classical Be stars.

\section{Main physical differences between $\mathrm{HAe}$ and $\mathrm{HBe} /$ classical Be stars}

As a consequence of the results discussed above, we need to study the main differences between Herbig Ae and Herbig Be/classical Be stars. The volume of ionized hydrogen around these stars decreases with decreasing effective temperature and luminosity. Similarly, the amount of neutral hydrogen increases with decreasing effective temperature, but this trend is perhaps very much altered by the presence of strong winds and chromospheres in the Herbig stars. The projected rotational velocities decrease only slightly with decreasing effective temperature. The hydrogen emission region is more disk-like in the $\mathrm{HBe} /$ classical Be stars than in HAe stars, in which a more spherically symmetric emission region seems to be present.

If magnetic fields are present, we may expect a magneto-centrifugal force to control the mass ejection mechanism. Such a force can only work on conducting, ionized gas, with rapid rotation and a magnetic field as major ingredients. For a given magnetic field, the efficiency of such a potential mechanism decreases towards later spectral types because of the smaller volume of conducting material. However, we understand that this approach is highly phenomenological and we expect in reality a competition among different mass loss mechanisms.

\section{Proposed framework for mass loss}

At this stage we would like to propose a framework which can be used to base a general picture for mass loss among the $\mathrm{O}-, \mathrm{B}$ - and A-type stars. In the 
O-type stars, mass loss is dominated by radiation pressure. The efficiency of this mechanism decreases strongly with effective temperature. Among the early B-type stars a weak magnetic field combined with rapid rotation is able to accelerate ionized material (the magneto-centrifugal acceleration, MCA), leading to a slow, dense, outflow in the equatorial plane. The peak frequency distribution at spectral type B1 can be understood as a result of the decreasing efficiency of radiation pressure (which tends to remove the equatorial disk) and the increasing efficiency of the MCA mechanism. The MCA mechanism decreases in efficiency towards late B stars. However, the presence of a magnetic field allows the possibility of heating by magneto-hydrodynamic waves thought to be responsible for the mass loss around Herbig Ae stars.

\section{References}

Balona, L., Böhm, T. 1999, MNRAS, submitted

Böhm T., Catala C., 1995, A\&A, 301, 155

Donati, J.-F., Semel, M., Carter, B.D., Rees, D.E. Cameron, A.C. 1997, MNRAS291, 658

Hillenbrand, L.A., Strom, S.E., Vrba, F.J. Keene, J., 1992, ApJ397, 613

Lignières, F., Catala, C., Mangeney, A. 1996, A\&A314, 465

Quirrenbach, A., Bjorkman, K.S., Bjorkman, J.E., Hummel, C.A., Buscher, D.F., Armstrong, J.T., Mozurkewich, D., Elias, N.M.II., \& Babler, B.L. 1997, ApJ, 479, 477

Tout, C.A., Pringle, J.E. 1995, MNRAS272, 528

Vigneron, C., Mangeney, A., Catala, C., Schatzman, E. 1990, Solar Phys., 128, 287

\section{Discussion}

V. Airapetian: How would you expect/explain Alfvenically driven winds in Herbig Be stars with no surface convection?

T. Böhm: It is true that stars earlier than approximately F0 should not have, according to theoretical models, subphotospheric convection. However, in recent years several authors (Vigneron et al. 1990, Lignières et al. 1996, Tout \& Pringle) suggested mechanisms based on differential rotation of subphotospheric layers induced by the breaking of a stellar wind. this differential rotation induces a turbulence layer which might substitute the role of convection in the classical solar type dynamo mechanism and lead by this way to generation of magnetic fields. 